[5] R. Godement, Théorèmes tauberiens et théorie spectrale, Ann. Sei. Ecole Norm. Sup. (3) 64 (1947), p. 119 - 138.

[6] A. Zygmund, Trigonometrical series, Warsaw 1935.

[7] L. Schwartz. Théorie des distributions, II, Paris 1951.

[8] I. Kaplansky, Primary ideals in group algebras, Proe. Nat. Acad. Sei. U. S. A. 35 (1949), p. 133-6.

[9] L. Loomis, Abstract Harmonic Analysis, New York 1953.

[10] A. Weil, L'Integration dans les groupes topologiques et ses applications, Paris 1953.

Reçu par la Rédaction le 1. 8. 1959

\section{Ergodische Funktionale und individueller ergodischer Satz}

von

\section{S. GEADYSZ (Wrocław)}

$(S, \mathfrak{B}, m), m(S)=1$, sei ein festgesetzter Maßraum und $T$ eine meßbare nichtsinguläre Transformation von $S$ in $S$ (es ist also $T^{-1} B \in \mathfrak{Z}$, wenn $B \in \mathfrak{Z}$, und $m\left(T^{-1} B\right)=0$, wenn $\left.m(B)=0\right)$. Der Körper von meßbaren und $T$-invarianten Mengen soll mit $\mathfrak{B}_{T}$ oder genauer mit $\mathfrak{B}_{T}(m)$ bezeichnet sein $\left(B \in \mathfrak{B}_{T}\right.$ wenn $B \epsilon \mathfrak{B}$ und $\left.m\left(T^{-1} B \div B\right)=0\right)$, und der Raum von linearen Kombinationen der charakteristischen Funktionen der.Mengen aus $\mathfrak{B}$ mit $X(\mathfrak{B})$.

Es ist bekannt, daß man die Voraussetzung der Invarianz des Maßes in dem individuellen ergodischen Satze weit schwächen kann [2], [3]. Für die individuelle Konvergenz $m$-fast überall (weiter auch $\mathrm{m}$-f. ü. oder $[m]$ bezeichnet) genügt es z. B., wenn es ein solches $K$ gibt, daß

$$
\lim _{n} \frac{1}{n} \sum_{k=0}^{n-1} m\left(T^{-k} B\right) \leqslant K m(B), \quad B \in \mathfrak{Z} .
$$

Ebenso, kann man solche Voraussetzungen durch andere ersetzen, z. B. durch starke Konvergenz der ergodischen Mitteln

$$
f_{n}(s)=\frac{1}{n} \sum_{k=0}^{n-1} f\left(T^{k} s\right)
$$

in $L^{r}(m), r \geqslant 1$ [2].

In dieser Arbeit ist die Bedeutung aufgeklärt, welche bei solchen Sätzen die hier ergodisch genannten Funktionale, besitzen. Die Existenz eines solchen Funktionals auf $L^{1}(m)$ ist mit der f. ü.-Konvergenz von $f_{n} \rightarrow f^{*} \epsilon L^{1}(m)$ gleichbedeutend. Daraus folgt sofort, daß die individuelle f. ü.-Konvergenz nicht nur eine Konsequenz der starken, sondern auch der schwachen und dabei nach einem einzigen Funktionale $\int \cdot d m$ ist. Wie bekannt [2], umgekehrt verursacht die f. ü. -Konvergenz noch nicht die starke. Es entsteht die Frage ob dann wenigstens die Konvergenz der Integrale $\int f_{n} d m$ folgt. Dann wäre $\lim \int f_{n} d m$ ein natürliches ergodisches Funktional. Beispiel 3 (in 3 ) zeigt, daß im allgemeinen dies auch nicht stattfindet.

studia Mathematica XIX 
Anstatt der Maße $m\left(T^{-k} B\right), k=0,1, \ldots$, ist es hier bequemer entsprechende ergodische Funktionale auf $X(\mathfrak{B})$ zu betrachten. Dem eben zittierten Falle entspricht das ergodische Funktional

$$
\varlimsup \lim \int f_{n} d m, \quad f \in X(\mathfrak{Z}) \text {. }
$$

Es ergibt sich, daß die individuelle Konvergenz der ergodischen Mitteln gleichbedeutend mit der Existenz und Stetigkeit irgendeines ergodischen Funktionals auf $X(\mathfrak{B})$ ist.

1. Jetzt gehen wir zur exakten Formulierung der Resultate über.

$X$ sei ein linearer Raum von meßbaren Funktionen, die $\bmod m$ identifiziert sind. Es wird immer vorausgesetzt, daß $X(\mathfrak{B}) \subset X$, wo $X(\mathfrak{Z})$ der Raum von linearen Kombinationen der meßbaren charakteristischen Funktionen ist. Mit $X^{+}$wird der positive Kegel von nichtnegativen Funktionen aus $X$ bezeichnet.

Das Funktional $p$, definiert auf $X^{+}$, soll ergodisch heißen, wenn es konvex:

$$
\begin{gathered}
p(t \cdot f)=t \cdot p(f), \quad t \geqslant 0 \\
p(f+g) \leqslant p(f)+p(g)
\end{gathered}
$$

monoton:

$$
0 \leqslant p(f) \leqslant p(f+g)
$$

invariant:

$$
p(T f)=p(f), \quad T f(.)=f(T .),
$$

und konservativ:

$$
p(f)=\int f d m \text { für } f \in X^{+}\left(\mathfrak{Z}_{T}\right),
$$

ist.

Es gibt eine strenge Beziehung zwischen der Existenz eines solchen Funktionals in manchen Funktionalräumen und der Existenz eines $T$-invarianten Maßes. Für die in der Ergodentheorie wichtigsten Räume $L^{r}(m)$ gilt nämlich

SATz 1. Ist $1<r<\infty$, so sind I, II und IV äquivalent und ziehen III nach sich, dagegen sind für $r=1$ alle vier folgenden Thesen äquivalent:

I. Es existiert auf $L^{r+}(m)$ ein ergodisches Funktional.

II. Es existiert auf $X^{+}(\mathcal{B})$ ein ergodisches Funktional $p$ und ein solches $K, d a \beta$

$$
p(f) \leqslant K .\|f\|_{r} .
$$

III. Es existiert auf $\mathfrak{Z}$ ein invariantes $\sigma$-Maß $\mu$ konservativ auf $\mathfrak{Z}_{T}\left(^{\left({ }^{1}\right)}\right.$ und ein solches $K$, da. $\beta$

$$
\mu(B) \leqslant K \cdot[m(B)]^{1 / \boldsymbol{r}}, \quad \boldsymbol{B} \in \mathfrak{B} .
$$

() d. h. $\mu=m$ auf $\mathfrak{B}_{T}(m)$
IV. Für jede $f \in L^{s}(m), s \geqslant r$, existiert $m-f$. $\ddot{u} . \lim f_{n}=f^{*} \epsilon_{\epsilon} L^{s / \mathbf{r}}(m)\left(^{2}\right)$. Beweis dieses Satzes, wie auch anderer Sätze, ist in 2 gegeben.

Wir machen noch die Bemerkung, daß man in IV im allgemeinen nicht erwarten kann, daß $f^{*} \epsilon L^{s}(m)$ anstatt in $L^{s / r}(m)$, wie es Beispiel 1 zeigt. Ebenso, für $r>1$, ist III nicht mit anderen Thesen äquivalent (Beispiel 2).

Für den Raum der beschränkten Funktionen $L^{\infty}(m)$ ist Satz 1 schon falsch, da dort immer ein ergodisches Funktional existiert (z. B. $\varlimsup T^{n} f d m$ ), dagegen ist im allgemeinen IV nicht wahr. Und so in $L^{\infty}$ folgt IV nicht aus I. Aber diese Folgerung besteht, wenn das ergodische Funktional im gewissen Sinne stetig, oder auf einem mehr als $L^{\infty}$ umfangreichem Raume erklärt ist.

Das ergodische Funktional $p$ ist m-stetig, wenn

$$
p\left(f_{n}\right) \rightarrow 0, \quad \text { für } \quad f_{n} \downarrow 0[m], \quad f_{n} \in X^{+}(\mathfrak{Z}) .
$$

Offenbar, wenn das Funktional $p$ die Bedingung (1) erfüllt, so ist es $m$-stetig.

Ein $Z(m)$ Raum ist ein linearer $F$-Raum mit der im allgemeinen nicht homogenen Norm $\|$.$\| , dessen Elemente 2$-meßbare Funktionen identifiziert $\bmod m$ sind. Es wird vorausgesetzt, daß $X(\mathfrak{B}) \subset Z(m), \operatorname{da} \beta|f| \epsilon Z$, wenn $f \in Z$ und $\||f|\|=\|f\|$, daß der Kegel $X^{+}$von nichtnegativen Funktionen abgeschlossen ist und daß

$$
\left\|f_{n}\right\| \rightarrow 0, \quad \text { wenn } \quad 1 \geqslant f_{n} \downarrow 0[m] .
$$

Eine Operation $\varphi$, die $Z^{+}(m)$ in den Raum von meßbaren Funktionen abbildet, heißt ergodisch, wenn sie konvex, monoton, invariant und konservativ ist. Dies letzte bezeichnet, daß $\varphi(f) \in L^{1}(m)$, wenn $f \in X^{+}(\mathfrak{Z})$ und daß $\varphi(f)=f[m]$, wenn $f \in X^{+}\left(\mathfrak{B}_{T}\right)$.

SATz 2. Die Thesen $\mathrm{II}^{\prime}$, $\mathrm{III}^{\prime}$ und $\mathrm{IV}^{\prime}$ sind äquivalent $\left(^{3}\right)$ und folgen aus $I^{\prime}$

$\mathrm{I}^{\prime}$. Es existiert auf $Z^{+}(m)$ ein ergodisches Funktional oder eine ergodische Operation.

II'. Es existiert auf $X^{+}(\mathfrak{B})$ ein $m$-stetiges ergodisches Funktional. III'. Es existiert anf $\mathfrak{Z}$ ein invariantes und konservatives auf $\mathfrak{B}_{T}$ $\sigma-M a \beta \mu<m$.

$\mathrm{IV}^{\prime}$. Für jede $f \epsilon L^{\infty}(m)$ existiert $m$-f. ü. $\lim f_{n}$.

$\left(^{2}\right)$ Aus I oder II folgt IV für jedes $s \geqslant r$, dagegen umgokehrt, wenn man andere Thesen aus IV bekommen will, genügt es in IV nur $s=r$ zu setzen.

(') Daß III' aus IV' folgt, hat C. Ryll-Nardzewski bemerkt. 
2. Beweise. Huffssatz 1. Gibt es auf $X^{+}$ein ergodisches Funktional $p$, so gibt es auf dem ganzen $X$ ein lineares ergodisches (also positives) Funktional $F$ mit

$$
F^{\prime}(f) \leqslant p(f) \text { für } f \geqslant 0 .
$$

Beweis. Das auf dem ganzen $X$ definierte Funktional $q(f)=$ $=p\left(f^{+}\right)\left({ }^{4}\right)$ ist offenbar invariant und konvex, was leicht aus der Kon vexität und Monotonität von $p$ folgt. Da, wegen der Konservativität,

$$
F(f)=\int f d m \leqslant q(f) \quad \text { auf } \quad X\left(\mathfrak{B}_{T}(m)\right),
$$

so kann man das lineare Funktional $F$ aus $X\left(\mathcal{B}_{T}(m)\right)$ zum ganzen $X$ linear fortsetzen, wobei die Invarianz und die Bedingung

$$
F(f) \leqslant q(f)
$$

gültig bleiben ([5], Satz 15)

Es bleibt nur zu zeigen, daß $F(f) \geqslant 0$, wenn $f \geqslant 0$, was aus der Monotonität von $q$ folgt: für $f \geqslant 0$ ist

$$
-F(f)=F(-f) \leqslant q(-f)=p(0)=0 .
$$

HسFssatz 2. Ist $p$, im Hilfssatz 1 , ein m-stetiges ergodisches Funktional, so ist $\mu(B)=F\left(\chi_{B}\right)\left({ }^{5}\right)$ ein invariantes, konservatives $\left({ }^{6}\right) \sigma$-Ma $\beta$ mit

$$
\mu(B) \leqslant p\left(\chi_{B}\right) .
$$

Beweis. Aus dem Hilfssatz 1 folgt unmittelbar, daß $\mu$ ein endlich additives $\mathrm{Ma}$ ist, das außerdem alle verlangte Eigenschaften besitzt. Es genügt also zu zeigen, daß $\mu$ ein $\sigma$-additives, also stetiges Maß ist, was sofort aus (4) und aus der Stetigkeit von $p$ folgt.

HIFSSATZ 3. $X$ sei ein abstrakter linearer F-Raum ([1], S. 35), $X^{+} \subset X$ ein abgeschlossener positiver Kegel und $p$ ein Funktional auf $X^{+}$. Gilt für $p$ (auf $X^{+}$)

$$
\begin{gathered}
p(x / n) \underset{n}{\rightarrow}, \\
p(x+y) \geqslant p(x) \geqslant 0,
\end{gathered}
$$

so gilt auch

$$
p\left(x_{n}\right) \underset{n}{\rightarrow} 0, \quad \text { wenn } \quad\left\|x_{n}\right\| \underset{n}{\rightarrow} 0, \quad x_{n} \in X^{+}
$$

(4) $f^{+}=f^{+}(s)=\max [0, f(s)]$.

(5) $\chi_{B}$ bezeichnet die oharakteristisehe Funktion der Menge $B$, also $\chi_{B}(8)=1$ wenn $s \in B$ und 0 sonst.

( $\left.{ }^{6}\right) \operatorname{Siehe}\left({ }^{1}\right)$.
Beweis. Wäre (8) nicht wahr, so gäbe es eine solche Folge $x_{k} \in X^{+}$, daß

$$
\left\|x_{k}\right\| \underset{\vec{k}}{\rightarrow} 0, \quad \text { wobei } \quad p\left(x_{k}\right) \geqslant a>0 .
$$

Da $\left\|n x_{k}\right\| \rightarrow \vec{k} 0, n=1,2, \ldots$, könnte man eine Teilfolge (weiter auch mit $x_{k}$ bezeichnet) mit $\sum_{n=1}^{\infty}\left\|n x_{n}\right\|<\infty$ finden. Das zieht nach sich die Konvergenz jeder der Reihen $y_{N}=\sum_{n=N}^{\infty} n x_{n}, N=1,2, \ldots$, wobei $y_{N} \in X^{+}$, da $X^{+}$abgeschlossen ist.

$p\left(y_{1}\right)$ hat also einen Sinn und dabei ergibt sich, aus $(6), p\left(y_{1} / N\right) \underset{N}{ } 0$. Nach (7) und (9) wäre aber

$$
p\left(y_{1} / N\right)=p\left(\frac{1}{N} \sum_{n=1}^{N-1} n x_{n}+x_{N}+y_{N+1} / N\right) \geqslant p\left(x_{N}\right) \geqslant a>0 .
$$

Dieser Widerspruch bestätigt (8).

HILFSSATZ 4. $p$ sei ein homogenes Funktional, erklärt auf einem abgeschlossenen positiven Kegel $X^{+}$eines abstrakten Banachschen Raumes $X$. Ist $p$ auch monoton, gilt also für $p$ auch (7), so gibt es eine solche Konstante $K$, $d a \beta$

$$
p(x) \leqslant K .\|x\|, \quad x \in X^{+} .
$$

Beweis wie üblich bei solchen Sätzen: im Gegenfalle gäbe es eine Folge $x_{n} \in X^{+}$mit $p\left(x_{n}\right) \geqslant n$. $\left\|x_{n}\right\|$. Für $y_{n}=x_{n} /\left\|x_{n}\right\|$ wäre dann $y_{n} / n \rightarrow 0$ und gleichzeitig

$$
p\left(y_{n} / n\right)=p\left(y_{n}\right) / n \geqslant 1
$$

was bereits mit Hilfssatz $3 \mathrm{im}$ Widerspruch steht.

Beweis des Satzes 1 . I $\rightarrow$ II nach Hilfssatz 4.

II $\rightarrow$ III. Nach (1) ist das Funktional $p m$-stetig und darum (Hilfssatz 2) existiert auf $\mathfrak{B}$ das verlangte $\mathrm{Ma} \beta \mu$, das wegen (5) und (1) die Ungleichung (2) erfüllt.

II $\rightarrow$ IV. Das Funktional $F$ des Hilfssatzes 1, definiert auf $X=X(\mathfrak{B})$, besitzt folgende Eigenschaften:

$1^{\circ}$ aus (1) und $(4): F(|f|) \leqslant p(|f|) \leqslant K$. $\|f\|_{r}$;

$2^{\circ}$ aus der Definition von $\mu$ (Hilfssatz 2) folgt $F(f)=\int f d \mu$.

Da aber $X(\mathfrak{B})$ dicht ist, gilt die Ungleichung

$$
\int|f| d \mu \leqslant K .\|f\|_{r}, \quad f \in L^{r}(m),
$$

allgemein. Darum, wenn $f \in L^{s}(m)$, oder, was gleichwertig ist, $f^{s / r} \in L^{r}(m)$, so $f^{s / r} \in L^{1}(\mu)$. 
Die Grenze $\lim f_{n}=f^{*}$ existiert also $\mu$-f. ü. und dabei $f^{*} \epsilon L^{s ; \gamma^{*}}(\mu)$. Da $T$ eine nichtsinguläre Transformation in bezug auf $m$ ist, so ist $f^{*} \mathfrak{B}_{T}(m)$-meßbar. So existiert $\lim f_{n}$ auch $m$-f. ii. und $f^{*} \in L^{s / r}(m)$, weil $m=\mu$ auf $\mathfrak{B}_{T}(m)$ ist.

$\mathrm{IV} \rightarrow \mathrm{I}$. Wenn $f \epsilon L^{r}(m)$, so $f^{*} \epsilon L^{1}(m)$ und $p(f)=\int f^{*} d m$ ist trivial ein ergodisches Funktional.

III $\rightarrow$ II. Wie vorher, wobei (1), für $r=1$, aus (2) folgt.

Beweis des Satzes 2. $\mathrm{I}^{\prime} \rightarrow \mathrm{II}^{\prime}$. Es sei $p$ ein ergodisches Funktional auf dem abgeschlossenen Kegel $Z^{+}(m)$. Aus Hilfssatz 3 folgt

$$
p\left(f_{n}\right) \rightarrow 0, \quad \text { wenn } \quad\left\|f_{n}\right\| \rightarrow 0, \quad f_{n} \in Z^{+} .
$$

Jetzt sei $B_{1} \supset B_{2} \supset \ldots, \bigcap B_{n}=0$. Dann, wegen (3) ist $\left\|\chi_{B_{n}}\right\| \rightarrow 0$, also auch $p\left(\chi_{B_{n}}\right) \rightarrow 0$, was die verlangte Stetigkeit von $p$ gibt.

Wenn dagegen auf $Z^{+}$eine ergodische Operation $\phi$ erklärt ist, so führt man auf $X^{+}(\mathfrak{B})$ ein Funktional $p(f)=\int \varphi(f) d m$ ein, das offenbar ergodisch ist. Es genügt zu zeigen, daß $p m$-stetig ist.

Das Hilfsfunktional $q(f)=\int \operatorname{arctg} \varphi(f) d m$ ist bereits auf dem ganzen $Z^{+}$definiert. Es ist zwar nicht homogen, aber monoton und erfüllt (6). Hilfssatz 3 ist also anwendbar, woraus die Stetigkeit von $q$ folgt; genauer, daß

$$
q\left(f_{n}\right) \rightarrow 0, \text { wenn }\left\|f_{n}\right\| \rightarrow 0 \text { und } f_{n} \geqslant 0 .
$$

Daraus folgt die asymptotische Konvergenz $\varphi\left(f_{n}\right) \underset{\text { as }}{\rightarrow} 0$.

Wenn also $B_{1} \supset B_{2} \supset \ldots, \cap B_{n}=0$, so ist (nach (3)) $\left\|\chi_{B_{n}}\right\| \rightarrow 0$ und daher $q\left(\chi_{B_{n}}\right) \rightarrow 0$, was schließlich $\varphi\left(\chi_{B_{n}}\right) \rightarrow 0$ gibt. Dies und die Monotonität von $\varphi$ gibt weiter

$$
p\left(\chi_{B_{n}}\right)=\int \varphi\left(\chi_{B_{n}}\right) d m \rightarrow 0,
$$

also die $m$-Stetigkeit von $p$

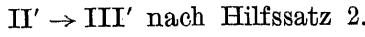

$\mathrm{III}^{\prime} \rightarrow \mathrm{IV}^{\prime}$ wie üblich: aus der Konservativität folgt, $\operatorname{da} \beta \lim f_{n}$, der sicher $\mu$-f. ü., auch $m$-f. ü. existiert.

$\mathrm{IV}^{\prime} \rightarrow \mathrm{III}^{\prime}$. Für jedes $B \in \mathfrak{B}$ existiert die Grenze

wo

$$
\mu(B)=\lim \mu_{n}(B),
$$

$$
\mu_{n}(B)=\int \frac{1}{n} \sum_{k=0}^{n-1} \chi_{B}\left(T^{-k} s\right) d m
$$

ist. Dieses $\mu$, als Grenze von absolut stetigen und $\sigma$-additiven Maßen ist auch ein absolut stetiges und $\sigma$-additives $\mathrm{Maß}$ [4], das offenbar invariant ist.
$\mathrm{III}^{\prime} \rightarrow \mathrm{II}^{\prime}$ trivial, weil $\int f d \mu$ alle verlangten Eigenschaften besitzt.

3. Beispiele. 1. In IV (Satz 1) ist im allgemeinen nicht $f^{*} \in L^{s}(m)$, wenn $f \epsilon L^{s}(m)$, wie es das folgende Beispiel zeigt.

Es $\quad$ sei $S=\left\{s_{1}, s_{2}, \ldots\right\}, T s_{2 k-1}=s_{2 k}, \quad T s_{2 k}=s_{2 k-1}, \quad k=1,2, \ldots$ und $m\left(s_{2 k-1}\right)=2^{-k}-2^{-3 k / 2}, m\left(s_{2 k}\right)=2^{-3 k / 2}$. Hier besteht $\mathfrak{Z}_{T}$ aus Kombinationen von Mengen $\left\{s_{2 k-1}, s_{2 k}\right\}$ und das konservative und invariante $\mathrm{Maß}$ ist $\mu\left(s_{2 k-1}\right)=\mu\left(s_{2 k}\right)=2^{-k-1}$. Daher

$$
h=\frac{d \mu}{d m}=\left\{\begin{array}{cll}
1 / 2\left(1-2^{-k / 2}\right)<2, & \text { wenn } & s=s_{2 k-1}, \\
2^{k / 2-1}, & \text { wenn } & s=s_{2 k},
\end{array}\right.
$$

und $\int h^{2} d m<\infty$.

$$
\text { Ist } f \in L^{2}(m) \text {, so gilt }
$$

$$
\int|f| d \mu=\int|f| \cdot h d m \leqslant\|f\|_{2} \cdot\|h\|_{2}<\infty
$$

und $f \in L^{1}(\mu)$. Deshalb ist das Funktional $p(f)=\int f d \mu$, wegen der Konservativität von $\mu$, ein ergodisches Funktional auf $L^{2}(m)$. Nach Satz 1 sind also I-IV für $r=2$ erfüllt.

Ist $f \in L^{2}(m)$, so ist $f^{*} \epsilon L^{1}(m), s=r=2$, was man auch leicht unmittelbar prüfen kann, aber nicht unbedingt $f^{*} \in L^{2}(m)$, wie es die Funktion

$$
f(s)=\left\{\begin{array}{lll}
0, & \text { wenn } & s=s_{2 k-1}, \\
2^{k / 2}, & \text { wenn } & s=s_{2 k},
\end{array}\right.
$$

zeigt. Für diese gilt nämlich offenbar $\int f^{2} d m=\sum 2^{k} \cdot 2^{-3 k / 2}<\infty$ und gleichzeitig $\int f^{* 2} d m \geqslant \sum 2^{k-2} \cdot m\left(s_{2 k-1}\right)=\infty$.

2. Wir werden zeigen, daß im Satz 1 , für $r=2$, III mit anderen Thesen nicht äquivalent ist.

$S$ und $T$ seien wie im Beispiel 1. Das Maß $m$ wird folgendermaßen definiert: $m\left(s_{2 k-1}\right)=2^{-k}-2^{-2 k}, \quad m\left(s_{2 k}\right)=2^{-2 k}$, so daß wie vorher $\mu(s)=2^{-k-1}$, wenn $s=s_{2 k-1}$ oder $s_{2 k}$.

Offenbar gilt $\mu(B) \leqslant[m(B)]^{1 / 2}$ und so ist die Bedingung III im Satz 1 für $r=2$ erfüllt. Dagegen ist IV nicht erfüllt, da es eine Funktion $f \in L^{2}(m)$ gibt, für die $f^{*} \notin L^{1}(m)$. Eine solche is z. B

$$
f(s)=\left\{\begin{array}{lll}
0, & \text { wenn } & s=s_{2 k-1}, \\
2^{k} / k, & \text { wenn } & s=s_{2 k} .
\end{array} .\right.
$$

Dann hat diẹ Grenzfunktion die Gestalt $f^{*}(s)=2^{k-1} / k$ für $s=s_{2 k-1}, s_{2 k}$ und es gilt

$$
\int f^{*} d m \geqslant \sum \frac{2^{k-1}}{k} \cdot m\left(s_{2 k-1}\right)=\infty .
$$


3. Es wird das Beispiel einer Transformation $T$ gezeigt, für welche: $1^{\circ} T f \in L^{1}(m)$, wenn $f \in L^{1}(m), 2^{0}$ die Grenze $f^{*}=\lim f_{n}$ existiert überall für jede endliche Funktion, $f$ und $3^{0} f^{*} \epsilon L^{1}(m)$, wenn $f \in L^{1}(m)$ und dabei Beispiel einer Funktion $f \in L^{1}(m)$ mit $\int T^{n} f d m \rightarrow \infty$.

Es sei $S$ eine abzählbare Menge verteilt auf Untermengen $S_{n}$ $n=1,2, \ldots$, jede mit $2^{n}+n+1$ Punkten $s_{n, 1}, s_{n, 2}, \ldots$ mit den Maßen

$$
m\left(s_{n, k}\right)=\left\{\begin{array}{ll}
2^{-(n+k+1)}, & \text { wenn } \\
2^{-(2 n+1)} & \text { sonst. }
\end{array} \leqslant n,\right.
$$

Es ist $m\left(S_{n}\right)=\sum_{k} m\left(s_{n, k}\right)=2^{-n}$, so daß $m(S)=\sum_{n} m\left(S_{n}\right)=1$. Die Transformation $T$ werde folgendermaßen definiert

$$
T s_{n, k}=\left\{\begin{array}{lll}
s_{n, k+1}, & \text { wenn } & 1 \leqslant k \leqslant 2^{n}+n, \\
s_{n, 1}, & \text { wenn } & k=2^{n}+n+1
\end{array}\right.
$$

Die invarianten Mengen sind Kombinationen von $S_{n}$ und das invariante und konservative $\mathrm{Ma} \mu$ ist leicht zu zeigen, nämlich

$$
\mu\left(s_{n, k}\right)=m\left(S_{n}\right) / 2^{n}+n+1=2^{-n} / 2^{n}+n+1 .
$$

Für jeden Punkt gilt

$$
\mu\left(s_{n, k}\right) / m\left(s_{n, k}\right) \leqslant \mu\left(s_{n, k}\right) / m\left(s_{n, n}\right) \leqslant 2
$$

und darum auch allgemein $\mu(B) \leqslant 2 m(B)$.

Daraus folgt sofort $f^{*} \epsilon L^{1}(m)$, wenn $f \in L^{1}(m)$. Denn aus der Konservativität folgt $f \in L^{1}(\mu)$ und daher $\int f^{*} d m=\int f^{*} d \mu=\int f d \mu<\infty$. Weiter, da $m\left(T^{-1} s_{n, k}\right) \leqslant 2 m\left(s_{n, k}\right)$, so allgemein $m\left(T^{-1} B\right) \leqslant 2 m(B)$ und $T f \in L^{1}(m)$ zusammen mit $f$.

Die folgende Funktion gibt das verlangte Beispiel:

$$
f(s)=\left\{\begin{array}{cc}
n .2^{n} & \text { wenn } \\
0 & \text { sonst. }
\end{array} \quad s=s_{n, n},\right.
$$

Offenbar ist $f \epsilon L^{1}(m)$, aber gleichzeitig

$$
\int T^{n-1} f d m \geqslant n \cdot 2^{n} \cdot m\left(s_{n, 1}\right)=n / 4,
$$

da $T^{n-1} s_{n, 1}$ gleich $s_{n, n}$ ist.

\section{Zitatennachweis}

[1] S. Banach, Théorie des opérations linéaires, Warszawa 1932.

2] N. Dunford and D. S. Miller, On the ergodic theorem, Trans. Amer. Math. Soc. 60 (1946), S. $538-549$.

[3] C. Ryll-Nardzewski, On the ergodio theorems (I), Studia Math. 12 (1951), S. 65.73.

[4] S. Saks, On some functionals, Trans. Amer. Math. Soc. 35 (1933), S. 549 - 556.

[5] R. J. Silverman, Means on semigroups and the Hahn-Banach extension property, ibidem 83 (1956), s. 222-237.

INSTYTUT MATEMATYCZNY POLSKIEJ AKADEMII NAUK

MATHEMATISCHES INSTITUT DER POLNISCHEN AKADEMIE DER WISSENSCHAFTEN

Reçu par la Rédaction le 12. 9. 1959 